\title{
Comunicación de la verdad en medicina: contribuciones desde una perspectiva psicológica
}

\author{
M Luz Bascuñán $\mathbf{R}^{\mathrm{a}}$.
}

\author{
Truth telling in medicine. \\ Psychological perspective
}

Truth telling in medicine is difficult and stressful. Medical training teaches to mitigate pain and suffering, but death and health deterioration causes feelings of frustration among physicians. Physicians tend to conceal bad news to avoid the suffering caused by such adverse information. However, veracity is an integral part of a respectful relationship and is the basis of confidence between patients and health providers. However, confusing truthfulness with a mere exposure of all the available information is an oversimplification of the problem. Therefore the real issue drifts from "how convenient is to communicate the truth" to "how to share the information with my patient" in a setting in which he can express his feeling and thoughts. Communicating the truth is an ethical imperative. The patient has the right to be informed and the physician must provide this information timely and taking into account the capacity, interest and emotional status of the patients. Therefore prudence, warmth and bioethical reasoning are required for a good medical practice (Rev Méd Chile 2005; 133: 693-8).

(Key Words: Communication barriers; Confidentiality; Duty to warn; Ethics, medical)

Recibido el 6 de octubre, 2004. Aceptado en versión corregida el 8 de marzo, 2005.

Departamento de Bioética y Humanidades Médicas, Facultad de Medicina, Universidad de Chile.

aPsicóloga, Master of Science (MSc).

T a "comunicación de la verdad", particularmen-

Lte cuando se trata de informar "malas noticias" es una situación estresante y difícil para los médicos. Por "mala noticia" entendemos cualquier información que altera negativamente la visión que el paciente tiene de su futuro. De allí que su impacto dependa de la brecha entre las

Correspondencia a: M Luz Bascuñán R. Avenida Salvador 486, Providencia. Fono-Fax: 2748855.

E-mail: mbascunan@med.uchile.cl expectativas del paciente y su condición, incluyendo una amplia variedad de casos (desde el resultado de un examen hasta un diagnóstico terminal).

El propósito de este trabajo es reflexionar sobre el proceso de comunicación de la verdad en medicina, sus dificultades y conveniencias, desde un punto de vista tanto ético como técnico, mostrando la necesaria vinculación (pero escasamente explicitada) entre la reflexión bioética y la literatura e investigación sobre comunicación y psicología médica. 
¿POR QUÉ ES DIFÍCIL COMUNICAR LA VERDAD?

Aun cuando los médicos constantemente deben informar a los pacientes sobre aspectos adversos de su salud, la literatura muestra enormes deficiencias para hacer esta tarea ${ }^{1}$. Se ha observado que la resistencia de los profesionales a informar malas noticias se debe a que esto genera gran stress, intensas emociones, sensación de responsabilidad por la noticia dada y temor a una evaluación negativa ${ }^{2}$. Esta actitud también está determinada por la vivencia del dolor y muerte del profesional y su capacidad para enfrentar los sentimientos de los demás ${ }^{3}$. La formación médica tradicionalmente ha enfatizado el proceso de sanar y reducir el sufrimiento, mientras que el deterioro y la muerte enfrentan al médico con las limitaciones de la medicina y sentimientos de frustración y culpa. Un importante vuelco es dado entonces por la noción de que "siempre hay algo que hacer" por el paciente. Las unidades de cuidados paliativos son aquellas que se proponen mejorar las condiciones de vida del paciente cuya enfermedad no responde a un tratamiento curativo. El objetivo, por lo tanto, no es evitar que el paciente muera, sino que ésta se produzca en las mejores condiciones posibles.

\section{¿QUIÉN ES RESPONSABLE DE COMUNICAR LA VERDAD?}

Tradicionalmente la responsabilidad de informar, así como del cuidado general del paciente, ha sido del médico tratante. Sin embargo, los cambios en la organización de la práctica de la medicina y el rápido desarrollo tecnológico han multiplicado el número de profesionales que ofrecen atención a un paciente $\mathrm{y}$, en muchos casos, no son los médicos quienes pasan más tiempo con él. En este contexto la comunicación y coordinación dentro del equipo de salud se ha transformado en un desafío ineludible de los centros asistenciales. El paciente y sus familiares frecuentemente consultan las mismas interrogantes a distintos profesionales. Con el fin de evitar la entrega de información disímil, el equipo de salud debe definir, de antemano, quienes se harán cargo de esta tarea.
¿POR QUÉ COMUNICAR IA VERDAD SI ÉSTA PRODUCE DOLOR?

El ocultamiento de la verdad al paciente es una postura que ha sido sostenida bajo el principio de no maleficencia. En esta línea se ha sugerido que la comunicación de la verdad puede dañar, atemorizar y angustiar al paciente desproporcionadamente y llevarle a rechazar tratamientos objetivamente recomendables. También se ha argumentado que no hay motivo para revelar la verdad cuando los pacientes no desean escucharla ni son capaces de comprender la información adecuadamente y que difícilmente puede informarse la "verdad" si es prácticamente imposible conocerla con certeza y precisión.

Sin embargo, esta línea de argumentación no ha estado sólidamente sustentada por la evidencia empírica ${ }^{4}$. En primer lugar, la literatura muestra que la mayoría de los pacientes desean saber la verdad y que los beneficios para un paciente adecuadamente informado son mayores que los riesgos temidos ${ }^{5,6}$. Diversos estudios, incluyendo aquellos con pacientes terminales, muestran los beneficios psicológicos que posee la comunicación de la verdad para el proceso emocional de aceptación de la enfermedad y muerte ${ }^{4}$. Además, actualmente los pacientes consultan disponiendo de mucha información, obtenida en internet $u$ otros medios no especializados, por lo que los esfuerzos deben orientarse a velar por la calidad de esta información más que a discutir la conveniencia de que se disponga de ella.

Frecuentemente son los familiares quienes solicitan que se oculte la verdad al paciente. Si bien esto tiene la intención de proteger al enfermo bajo el supuesto de que "si no sabe no sufre", también lo despoja del derecho a decidir qué hacer con su vida y su muerte. En estos casos, el paciente, de una u otra manera, sentirá que se le oculta algo y terminará perdiendo la confianza en el médico y en sus seres cercanos. Esta "conspiración del silencio" llevará al enfermo a aislarse y a vivir su enfermedad en soledad ${ }^{8}$.

La veracidad forma parte del respeto que merece toda persona y ésta es la base de toda relación de confianza. La mentira desconoce la dignidad intrínseca de todo enfermo, así como su libertad y unicidad. La mentira no permite que el paciente tenga una actitud realista ante su futuro 
ni sea capaz de dar un sentido trascendente al final de la vida. Sin embargo, el principio de veracidad es ambiguo en cuanto puede implicar el deber de no mentir (o engañar al paciente), como la obligación de ofrecer toda la información disponible. Siguiendo los criterios para el proceso de consentimiento informado, es importante informar sobre todas las circunstancias que inciden en la decisión a tomar (incluyendo el diagnóstico, pronóstico; y las características, propósitos y riesgos del tratamiento propuesto, de los tratamientos alternativos y de no seguir ningún tratamiento). Aún así, quedaría por determinar el grado de exhaustividad con que deben abordarse estos contenidos. Pero si reconocemos que la comunicación de la verdad no es un evento sino que un proceso en el tiempo, vemos que la cantidad de información ofrecida debe responder a los intereses y estado emocional del paciente, considerando qué y hasta dónde éste quiere saber en cada momento.

La veracidad, por lo tanto, no significa que deba forzarse al paciente a escuchar la verdad. Este puede legítimamente expresar su deseo a no ser informado, pero esto sólo es posible si se le ha ofrecido la posibilidad de recibir tal información.

Se ha sostenido también que la frase "comunicación de la verdad" denota un nivel de certeza del que carecen muchos diagnósticos, pronósticos y tratamientos. Entonces cabe preguntarse si las estimaciones médicas pertenecen al paciente tanto como su historia clínica o si el resguardo del médico permite exponer al paciente a información incierta.

En efecto, la comunicación de la verdad lleva a los profesionales a reconocer sus limitaciones y las de la medicina. Smith, describió los aspectos fraudulentos que tendría el acuerdo tradicional entre médicos y pacientes, en el cual la imagen idealizada de la medicina se basaría en expectativas irrealistas que terminan por frustrar a ambos. Es necesario reemplazar este trato por uno más realista donde los médicos acepten sus limitaciones y los pacientes comprendan que la enfermedad, la muerte y el dolor son parte de la vida y que la medicina es riesgosa e incierta ${ }^{9}$. En este sentido reconocer que la seguridad del profesional genera confianza en el paciente, no significaría negar la incertidumbre, sino que informar $\mathrm{y}$ enfrentar razonable y certeramente la misma ${ }^{10}$.
Por último, se ha sugerido que no tiene sentido informar a los pacientes si éstos no son capaces de comprender la compleja información médica necesaria para un discernimiento total de las alternativas de tratamiento. Sin embargo, este mismo argumento ha sido utilizado como evidencia de las deficiencias de los profesionales para comunicar la verdad ${ }^{11}$.

Paciente y profesional contribuyen con conocimiento esencial y específico para tomar las decisiones adecuadas. La contribución del paciente no tiene que ver con su manejo del conocimiento médico sino con el hecho que sólo él sabe cómo experimenta su enfermedad, cuáles son sus circunstancias sociales, hábitos y preferencias. El objetivo es, por tanto, que el paciente adquiera un cuadro realista sobre su estado y altemativas posibles. Como señaló Simón, en vez responsabilizar a los pacientes de su falta de comprensión, es necesario reflexionar críticamente sobre la manera en que los profesionales dan esta información ${ }^{12}$. Si se utiliza un lenguaje técnico, se da toda la información disponible en una ocasión y no se examina qué le interesa saber al paciente, se podrá confundir al más lúcido y calmado de los pacientes.

Actualmente existe acuerdo en que un paciente mentalmente competente tiene pleno derecho (ético, moral y legal) a cualquier información que desee sobre sí mismo. El reconocimiento del principio de autonomía genera entonces una nueva concepción del paciente (capaz y autónomo pese a estar enfermo), lo que a su vez, deviene en un nuevo modelo de relación asistencial cuyas bases son las actitudes deliberativas y de participación.

Sin embargo, el reconocimiento de los derechos del paciente no soluciona todos los problemas. La veracidad no puede confundirse con una apertura total o brutal. En este sentido el debate ha ido desde la pregunta por "cuán conveniente es comunicar la verdad" a la interrogante de "cómo compartir la información con el paciente" en un clima afectivo que le permita decirnos lo que siente y piensa.

\section{¿CómO COMUNICAR LA VERDAD?}

La comunicación de la verdad es un proceso en el cual se pueden distinguir los siguientes aspectos básicos ${ }^{13-15}$ : 
Preparación personal y lugar físico. Esto incluye la disposición de tiempo y calma, así como de claridad respecto a la información que debe ser abordada. Es importante disponer de un lugar físico privado donde médico y paciente puedan estar tranquilos y donde el paciente puede procesar esta información junto a sus familiares. Aunque esto parezca evidente, la mayoría de los centros asistenciales no disponen de este espacio y en muchos casos debe discutirse la condición del paciente en los pasillos o frente de otros pacientes.

Exploración de la información que maneja el paciente. La capacidad de escuchar del médico se pone a prueba para determinar cuánto ha entendido el paciente de la información que posee, cuán realista es su impresión y cuáles son sus fantasías y temores. Al examinar el grado de comprensión del paciente y el sentido que le atribuye al problema, no sólo se expresa preocupación, respeto y compromiso con sus ideas; sino que se comienza a determinar su deseo de recibir información.

Exploración del deseo de recibir información. Esto determinará el nivel de detalle con que la información será compartida. El paciente es quien ofrecerá las claves del momento apropiado para ser informado y discutir las alternativas de acción. Pero estas claves pueden ser muy sutiles y requieren de la sensibilidad del médico para atender e interpretar los mensajes verbales y no verbales del paciente. Es importante examinar activamente cuán involucrado el paciente desea estar. Primero porque las estimaciones de los profesionales suelen no ajustarse a los deseos de los pacientes y en segundo lugar porque a partir del hecho que algunos pacientes sean pasivos (callando, no solicitando información ni haciendo preguntas) no se desprende el que no deseen hacerlo ${ }^{16}$. La exploración de cuánto desea saber el paciente constituye una invitación a compartir información que debe hacerse con tacto y dejando claro que existen distintas preferencias, todas ellas válidas. Si el paciente rehuye (niega) ciertos temas o indica no querer ser informado, debe darse la seguridad de que si cambia de parecer existirán otras oportunidades para hacerlo.
Compartir la información con el paciente. En este punto el profesional conoce qué sabe, cuánto desea saber y el vocabulario que utiliza el paciente para expresarse. Esta será la línea de base 0 punto de partida para ofrecer información y comenzar la labor educativa. Esto se lleva a cabo progresiva y recurrentemente, reforzando los aspectos que maneja el paciente y las circunstancias favorables, atendiendo a sus reacciones emocionales y recogiendo la información no incorporada por él. La información es ofrecida de manera clara, realista y en términos accesibles. La clarificación es central en todo este proceso y se refiere a asegurar que ambos (profesional y paciente) den un mismo significado a los hechos.

Acoger los sentimientos del paciente. Esto determinará en última instancia el logro del proceso. El rango de reacciones emocionales es amplio y variable en el tiempo. Según Buckman hay tres criterios básicos para examinar las reacciones del paciente y estimar el mejor curso de acción: la aceptabilidad social (cuán esperable es la reacción de acuerdo a las normas culturales del paciente); adaptabilidad (si la reacción aumenta o alivia la aflicción del paciente) y reparabilidad (si existe alguna intervención -u otro profesional- que pueda ayudar al paciente en caso que la reacción aumente su aflicción) ${ }^{13}$.

Ante una noticia adversa es frecuente esperar una primera reacción de incredulidad. Como expresión de la negación muchas veces los pacientes señalan "nunca haber recibido información" o bien acuden a otros especialistas guardando la secreta esperanza de una "cura milagrosa". En estos casos, el paciente se rehusa inconscientemente a escuchar la información expresando que "no cree que sea real o que es un error" o bien actuando como si así fuera. La negación es un mecanismo defensivo ante eventos que nos sobrepasan. $\mathrm{Si}$ reconocemos su naturaleza protectora y sabemos que el bloqueo post-información impide que la persona recuerde lo que se le informa, evitaremos forzar al paciente y/o su familia a asumir prematuramente su situación y daremos información en forma reiterada tantas veces como sea necesario.

Cuando el paciente comienza a aceptar lo que le ocurre comúnmente se generan sentimientos de 
rebeldía, culpa y resentimiento. "¿Por qué a mí?, ¿qué he hecho para que me pase esto?". La rabia puede expresarse abierta o sutilmente y puede estar dirigida a uno o muchos objetivos como son: hacia sí mismo (expresándose en culpa), hacia la enfermedad, Dios, los familiares o el equipo de salud. En todos estos casos responder con juicios es poco favorable (aunque éstos intenten mitigar o mostrar la irracionalidad del sentimiento). Si comprendemos que el paciente necesita expresar su rabia para liberarse de ella, podremos evitar la frecuente tendencia a su personalización, vale decir, a vivirla como un ataque personal. Cuando ocurre esto es muy difícil poder sentir empatía y compasión.

Usualmente llega un momento en que el paciente se aflige profundamente, y necesita llorar. Los sentimientos de tristeza, como toda emoción fuerte, son altamente contagiosos. Frecuentemente es debido a la ansiedad que uno experimenta ante el dolor de otro, que tendemos a tranquilizarlo prematuramente impidiéndole expresarse. Contener las emociones del paciente no significa distraer su atención sino ser capaces de tolerar su sufrimiento de manera que pueda ir integrando su experiencia. Empatizar y contener al paciente implica conectarse con su estado emocional y responder a sus necesidades (y no a las propias) por ejemplo, evitando comunicar la propia desesperación cuando el otro necesita esperanza o aferrarse a la esperanza cuando el otro ya no la tiene.

Planificación y seguimiento. La comunicación de la verdad incluye el proceso de acompañamiento en el tiempo. Esto significa asegurar al enfermo que no se quedará solo y que se hará todo lo posible para ofrecerle las mejores condiciones sin importar cuán desfavorable sea el pronóstico. Al sintetizar, junto al paciente sus principales preocupaciones, en un plan de acción concreto, se establece un compromiso y se ofrece algún grado de control al paciente sobre lo que va a ocurrir con él.

Reconocimiento de las propias reacciones emocionales. Al igual que el paciente, el médico puede angustiarse, frustrarse, enrabiarse y ocupar distintos mecanismos para defenderse de estos senti- mientos. El auto-conocimiento del médico es necesario para manejar estas emociones en la relación con el paciente y contribuir a su propio auto-cuidado ${ }^{17}$. El trabajo personal del médico especialmente en relación a la polaridad omnipotencia-impotencia constituye un aspecto determinante desde el inicio y a lo largo de todo el proceso de comunicación de la verdad.

\section{COMENTARIO FINAL}

El intento de reducir el sufrimiento que provoca recibir información adversa y aquel que experimenta el profesional al tener que hacer tal revelación, son las dos dimensiones básicas tras el ocultamiento de la verdad al paciente.

$\mathrm{Si}$ bien el ocultamiento de la verdad socava la autonomía del paciente, la revelación de la verdad como la mera exposición de toda la información disponible es una simplificación del problema. Comunicar la verdad en medicina constituye un imperativo ético pero la determinación de la verdad y la oportunidad de su comunicación siguen siendo un juicio clínico. El médico realiza un juicio clínico de la capacidad del paciente para evaluar su situación así como acerca de qué y cuánta información éste desea y puede manejar en un momento determinado. Comunicar la verdad "brutalmente" puede ser tan dañino como la mentira. El paciente tiene el derecho de saber lo que le ocurre y el médico tiene el deber de informárselo, considerando su estado emocional y cognitivo.

La elaboración de guías o criterios generales para el proceso de comunicar la verdad así como para auto-cuidarse en esta tarea es de gran utilidad. Sin embargo, estas pautas siempre serán orientaciones generales, que deberán ser adaptadas a las características de la realidad sociocultural específica que se trate, así como interpretadas por el profesional poniéndose en juego su juicio y prudencia personal. Es decir, la disponibilidad de estos esquemas no asegura su aplicabilidad adecuada. Más aún en una época de crecientes regulaciones como es la nuestra, éstas pueden verse como un nuevo listado de más requerimientos, lo que terminaría por trivializar su significado y propósito fundamental. 
En este contexto no bastaría con establecer normas de acción sino que es necesario fundamentar e interiorizar los principios éticos que están a la base de tales cursos de acción. De allí que se enfatice la importancia de la formación de la persona del profesional, su calidad humana y raciocinio bioético con el fin de ofrecer al paciente el ejercicio libre de su auto-determinación en el marco de una relación de íntima confianza.

\section{REFERENCIAS}

1. UNGAR L Y COL. Breaking bad news: structures training for family medicine residents. Patient Education and Counseling 2002; 48: 63-8.

2. Baile W, Buckman R, Lenzi R, Glober G, Beale E, KuDELKA P. SPIKES-a six-step protocol for delivering bad news: application to the patient with cancer. Oncologist 2000; 5: 302-11.

3. LLYd M, BOR R. Communication skills for medicine. Londres: Editorial Churchill Livingston, 2001.

4. Sánchez M. Historia, teoría y método de la Medicina: Introducción al pensamiento médico. Madrid: Editorial Masson, 1998.

5. Simón P. Seminario-Taller: Consentimiento Informado. Serie de Documentos Académicos $\mathrm{N}^{\circ} 2$, División de Extensión Biomédica, Facultad de Medicina, Universidad de Chile, 1998.

6. Sumvan R, Menapace L, White R. Truth-telling and patient diagnoses. J Med Ethics 2001; 27: 192-7.

7. ANDREW J. Perspetives in Truth Telling, Etical Issues. En: Reich T, Ed. Enciclopedia of Bioethics. New York: MacMillan Library, 1995.

8. LLYy-WiшAms M. Breaking bad news to patients and relatives. BMJ 2002; 325: 511.
En vez de intentar negar las experiencias dolorosas que inevitablemente tiene la vida, es necesario obtener herramientas de comunicación, manejo de emociones, auto-conocimiento y auto-control con el propósito de ayudar al paciente y su familia y finalmente contribuir a la humanización de la práctica de la medicina.

9. Sмттн R. Why are doctors so unhappy? BMJ 2001; 322: 1073-4.

10. SHEPHERD D y COL. Effects of patient centredness and positive approach: Airing uncertainty can be positive. BMJ 2002; 324-543.

11. ARNold R, Lidz CH. Clinical aspects of informed consent. En: Reich T, Ed. Enciclopedia of Bioethics. New York: MacMillan Library, 1995.

12. Simón P, GuTiérRez J. Bioética para médicos. Med Clín 2001; 117: 99-106.

13. BucKman R. How to break bad news? A guide for health care professionals. Maryland, USA: The John Hopkins University Press, 1992.

14. BREWIN T. Relating to the relatives. Breaking bad news, communication and support. New York: Radcliffe Medical Press, 1996.

15. ARECHEDERRA JJ. Bioética, psiquiatría y derechos humanos. Madrid: International Marketing and communication SA, 1995.

16. BeISECKER AE, BeISECKER TD. Patient informationseeking behaviors when communicating with doctors. Med Care 1990; 28: 19-28.

17. SalinskY J, Sackin P. What are you feeling doctor? UK, Abingdon, Oxon: Radcliffe Medical press, 2000. 